\title{
Bound-State Energy of a Three-Boson System.
}

\author{
D. R. Avalos \\ Departamento de Física, Universidad Nacional de La Plata - La Plata
}

\author{
L. N. Epele $\left({ }^{*}\right)$ \\ $C E N$ de Saclay - Gif-sur-Yvette
}

M. A. GRegorio (*)

International Centre for Theoretical Physics - Trieste

(ricevuto il 14 Giugno 1973)

We are reporting here the results of some calculations made with Faddeev $\left({ }^{1}\right)$ equations for the simplest three-body system: the nonrelativistic three-boson system. Our aim is to study the $J=0$ bound-state energy spectrum of the system formed by three structureless alpha-particles $(\alpha)$ and compare it as a reference with the low-lying $J=0$ states of the nucleus ${ }^{12} \mathrm{C}\left({ }^{2}\right)$. The nuclear interaction between the alphas is represented by pair-wise $\left(V_{\alpha \alpha}\right)$ nonlocal separable potentials. We are, therefore, mainly concerned with the comparison of the different descriptions of the low-energy properties of the $3 \alpha$ system as obtained with different nuclear potentials $V_{\alpha \alpha}$.

To that end, the system under analysis is preferable to, say, the 3 -fermion system (3-nucleon system), since the involved angular-momentum algebra of the $3 \mathcal{N}$ system is absent here. The quantum numbers of the $3 \alpha$ system are simply obtained by adding the total (orbital) angular momentum of the $\alpha-\alpha$ pair to the orbital angular momentum of the spectator $\alpha$. We limit ourselves to the $J=0(l=0)$ three-body state and build the system by considering only $S$-wave $(\alpha-\alpha)$ states, neglecting higher partial waves. The subsystems $(\alpha-\alpha)$ are then described by the potential $V_{\alpha \alpha}$, whose parameters are calculated

(*) Fellow of the Consejo Nacional de Investigaciones Cientificas y Tecnicas, on leave of absence from Departamento de Física, Universidad Nacional de La Plata, La Plata.

(1) L. D. FADDEev: Sov. Phys. JETP, 12, 1014 (1961).

$\left(^{2}\right.$ F. AJZenberg-Selove and T. LaURitsen: Nucl. Phys., 11, 1 (1959).

( $\left.{ }^{3}\right)$ The $\alpha-\alpha S$-wave scattering phase shift was collected from the following articles (laboratory system energies): $a)(0.6 \div 3) \mathrm{MeV}:$ N. P. Hexdenburg and G. M. Temmer: Phys. Rev., 104, 123 (1956); b) $(3.84 \div 11.88) \mathrm{MeV}:$ T. A. Tombrello and L. S. Senhouse: Phys. Rev., 129, 2252 (1963); c) $(12.3 \div 23) \mathrm{MeV}:$ R. NImson, W. K. Jentsche, G. R. BRIGGs, R. O. KeRMAN and J. N. SNYDER: Phys . Rev., 109, $852(1958) ; d)(53 \div 77.55) \mathrm{MeV}$ : P. Darridlat, G. IGo, H. G. PUGH and H. D. HolmGReN: Phys. Rev., 137, B 315 (1965). 
by fitting the $S$-wave $(\alpha-\alpha)$ scattering phase shift $\left(\delta_{0}(E)\right)$ in the energy range (laboratory system) $(0.6 \div 77.0) \mathrm{MeV}$. Now, some interesting questions arise since, as is well known, $\delta_{0}(E)$ presents a very peculiar behaviour $\left({ }^{3}\right)$.

i) An $(\alpha-\alpha)$ resonance $\left({ }^{8} \mathrm{Be}\right)$ shows up at $E_{n}=94.5 \mathrm{keV}$, slightly above threshold for $\alpha-\alpha$ scattering. This point cannot be discussed by simply considering a nuclear potential; in fact, for energies up to $400 \mathrm{keV}$ the measured ratio of total to Mott scattering is exactly one $\left({ }^{3 a}\right)$, indicating that the Coulomb repulsion between the alphas is not disturbed by the nuclear force-up to that energy, of course.

ii) If we start at $E=0.6 \mathrm{MeV}, \delta_{0}(E)$ decreases monotonically with $E$ and changes sign at about $E_{c}=20 \mathrm{MeV}$. Unlike the first point, we can safely neglect the Coulomb interaction for this energy region (at laboratory energies of $0.6 \mathrm{MeV}$ the ratio to Mott scattering is already different from one $\left.\left({ }^{3 a}\right)\right)$ and look for a nuclear potential which in turn must combine an attractive and a repulsive part in order to reproduce the change in sign of $\delta_{0}(E)$.

Here we are concerned with the second point, neglecting the Coulomb interaction and using two different types of attractive-repulsive nuclear potentials: $a$ ) a rank-two of the Yamaguchi type $\left({ }^{4}\right)$ and $b$ ) a rank-one like that proposed by TABAKIN for the nucleon-nucleon problem $\left({ }^{5}\right)$. These potentials have the following analytical expressions:

a) Tamaguchi rank-two

$$
\begin{aligned}
V_{\alpha \alpha \alpha}^{(\mathrm{Y})}\left(\boldsymbol{p}, \boldsymbol{p}^{\prime}\right) & =\sum_{i=1,2} \lambda_{\alpha \alpha}^{(i)} g_{\alpha}^{(i)}(\boldsymbol{p}) g_{\alpha}^{(i)}\left(\boldsymbol{p}^{\prime}\right), \\
g_{\alpha}^{(i)}(\boldsymbol{p}) & =\frac{1}{p^{2}+\beta_{i}^{2}}
\end{aligned}
$$

b) Tabakin type

$$
\begin{aligned}
V_{\alpha \alpha}^{(\mathrm{T})}\left(\boldsymbol{p}, \boldsymbol{p}^{\prime}\right) & =\lambda_{\alpha \alpha}^{(\mathrm{T})} g_{\alpha}^{(\mathbf{T})}(\boldsymbol{p}) g_{\alpha}^{(\mathrm{T})}\left(\boldsymbol{p}^{\prime}\right) \\
g_{\alpha}^{(\mathbf{T})}(\boldsymbol{p}) & =\left(\boldsymbol{p}^{2}-p_{\varepsilon}^{2}\right)\left(\boldsymbol{p}^{2}+d^{2}\right) /\left[\left(\boldsymbol{p}^{2}+b^{2}\right)\left(\boldsymbol{p}^{4}+a^{4}\right)\right]
\end{aligned}
$$

In $a$ ) the attractive part is represented by the term with negative strength $\lambda_{\alpha \alpha}$, while $\beta_{i}$ measures the range of the corresponding term. In $b$ ) the attractive-repulsive character is obviously provided by the term $p^{2}-p_{c}^{2}$, where $p_{c}$ corresponds to $\delta_{0}\left(p_{e}\right)=0$. We refer to the original paper by TABAKIN for a further description of $V_{\alpha \alpha}^{(\mathrm{T})}$ : With potentials $(1)$ and (2) we fitted the "nuclear" $\left(^{*}\right) \delta_{0}(E)$. We also produce the same fit with a single rank-one potential of the Hulthén type in order to compare with other results. The result of the fit, together with the experimentally measured $\delta_{0}(E)$, are shown in Fig. 1 .

Let us now briefly deseribe the three-body problem. At the low energies in which we are interested and in the separable two-body potential approximation, the three-

${ }^{(4)}$ Y. Yamaguchi and Y. Yamaguchi: Phys. Rev., 95, 1635 (1954).

(5) F. TABAKIN: Phys. Rev., 174, 1208 (1968).

(*) Here "nuclear " means " $S$-wave phase shift without completely removing the Coulomb contribution ". This indicates the well-known fact that $\delta_{v}(E)$ appears in the full scattering amplitude in the following combination:

$$
f=f_{\text {nuc }}+f_{\text {Coul }}, \quad f_{\text {nuc }}=(2 i k)^{-1} \exp \left[2 i \sigma_{0}\right]\left[\exp \left[2 i \delta_{0}(E)\right]-1\right], \quad f_{\mathrm{Coul}}=(2 i k)^{-1}\left[\exp \left[2 i \sigma_{0}\right]-1\right],
$$

where $\sigma_{0}$ is the pure Coulomb phase shift. 


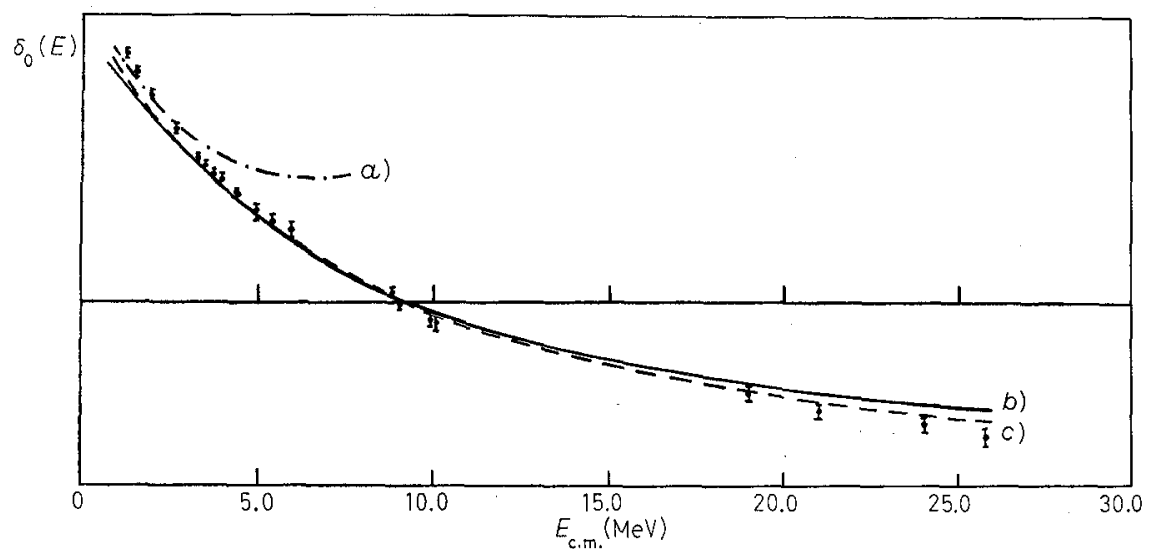

Fig. 1. - Experimental $S$-wave $\alpha-\alpha$ phase shifts, together with the fits obtained with $a$ ) Yamaguchi single term, b) Yamaguchi tank-two and c) Tabakin-type potential.

body system can be described by the Faddeev-Lovelace equations $\left({ }^{6}\right)$. These are multichannel Lippmann-Schwinger-type equations in which the number of channels, after angular-momentum reduction, is proportional to the number of separable terms contained in the two-body potentials. It is therefore clear that the three-body problem becomes simpler for an interaction of the type (2) than for a two-term potential like (1), and, actually, this is one of the reasons why the attractive-repulsive rank-one separable potentials were invented. For further details of the three-body equations in the separable approximation we refer to Lovelace's paper $\left({ }^{6}\right)$ and we only recall that the threebody bound-state energies are obtained by searching for the zeros of the Fredholm determinant of the corresponding homogeneous equation for the elastic $\left(\alpha,{ }^{8} \mathrm{Be}\right)$ scattering amplitude. This search was made by standard numerical procedures. Our twobody potential, as is natural for attractive or attractive-repulsive separable potentials $\left({ }^{7}\right)$, predicts a $2 \alpha$ bound state $\left({ }^{8} \mathrm{Be}\right)$ at an energy $E_{2 \alpha}$, and therefore our search for the $3 \alpha$ bound state was limited to $E_{3 \alpha}$ negative and $E_{3 \alpha} \leqslant E_{2 \alpha}$.

The results of these calculations are shown in Table I, where we also display all the two-body parameters. The result for model $O$ ) in Table I should not be taken seriously, since a single attractive term cannot give a unique set of parameters through the fit to $\delta_{0}(E)$. It must be said, however, that the fit was done without "completely removing " the Coulomb part, and, therefore, our model $C$ ) is not directly comparable with that of HaRRington $\left({ }^{8}\right)$. Some interesting information emerges from the comparison between $A$ ) and $B$ ) in Table $\mathrm{I}$. There is no excited state in model $A$ ), while model $B$ ) gives two $J=0$ states, as experimentally verified. The predicted ground state is, however, too low in both models $A$ ) and $B$ ). We can argue that by properly adding the Coulomb repulsion, the spectrum will shift enough in the right direction. According to ref. $\left({ }^{8}\right)$, the Coulomb energy of $3 \alpha$ is equal to $5.44 \mathrm{MeV}$, which is not enough to put theoretical and experimental results in a better agreement. However, the Coulomb repulsion will modify both models in the same way', since it depends on the asymptotic behaviour of the ${ }^{8} \mathrm{Be}$ wave function, which is the same for both models $A$ ) or $B$ ). Therefore, the excited state will still be absent in model $A$ ).

$\left(^{6}\right)$ C. Lovelade: Phys. Rev., 135, B 1225 (1964).

(7) G. C. Gmirardi and A. Rimini: Joum. Math. Phys., 5, 722 (1964),

$\left(^{8}\right)$ D. R. Harrington: Phys. Rev., 147, 685 (1966). 
TABLE I. - Two-body parameters and bound-state energies for the $J=0$ state of the $3 \alpha$ system. The energies are measured with respect to the $2 \alpha$ and $3 \alpha$ disintegration thresholds for two- and three-body systems, respectively. Our units are such that $m_{\alpha}=1, \hbar=1$ and $\lambda_{\mathrm{C}}=\hbar / m_{\alpha} c=0.052926 \mathrm{fm}$.

\begin{tabular}{|c|c|c|c|c|c|}
\hline & \multirow{2}{*}{\multicolumn{2}{|c|}{ Parameters }} & \multirow{2}{*}{$\begin{array}{l}E_{2 \alpha} \\
(\mathrm{MeV})\end{array}$} & \multicolumn{2}{|l|}{$E_{3 \alpha}(\mathrm{MeV})$} \\
\hline & & & & ground state & excited state \\
\hline $\begin{array}{l}\text { A) Yamaguchi } \\
\text { rank-two }\end{array}$ & $\begin{array}{l}\lambda_{1}=-1229.6 \\
\lambda_{2}=1091.6\end{array}$ & $\begin{array}{l}\beta_{1}=4.28 \\
\beta_{2}=3.9\end{array}$ & -16.9 & -24.5 & - \\
\hline $\begin{array}{l}B) \text { Tabakin } \\
\text { rank-two }\end{array}$ & $\begin{array}{lc}\lambda=-261.1, \\
d= & 0.86, \\
p_{c}= & 3.1\end{array}$ & $\begin{array}{l}a=2.4 \\
b=10.23\end{array}$ & -1.2 & -25.6 & -1.45 \\
\hline C) $\begin{array}{l}\text { Yamaguchi } \\
\text { single-term }\end{array}$ & $\lambda=-\quad 6.9$ & $\beta=2.57$ & -6.62 & -26.1 & -7.3 \\
\hline \multicolumn{4}{|c|}{$\begin{array}{l}\text { Experimental values for the low-lying } \\
J=0 \text { states of }{ }^{12} \mathrm{C}\end{array}$} & -7.28 & +0.376 \\
\hline
\end{tabular}

These results seem to represent another example of the different low-energy property description of a three-body system achieved by using either a rank-one or a rank-two attractive-repulsive separable potential. The first example is the three-nucleon system which we analysed in a previous work $\left({ }^{9}\right)$. At that time we found that the Tabakintype potential did not give as good a result as a rank-two potential. In the three-nucleon problem there are bound-state scattering data-the nucleon-deuteron scattering process-and a comparison between theory and experiment can therefore be made for the scattering energy region. This is not the case for the $\mathbf{3} \alpha$ system, nor for any of the simplest three-boson systems (three pions for instance $\left({ }^{10}\right)$ ), because a two-boson stable bound state, to be used as a target, camnot be found in Nature, and only the three-body bound-state region can be analy'sed and compared with experimental results. From the present analysis we can conclude that the rank-one type of potential gives a better description of the $3 \alpha$ bound-state spectrum than the Yamaguchi rank-two type. However, no general answer emerges from the present calculation to the question of which it is best to use in a three-body problem, and we are only able to say that the Tabakin potential is more suitable for the present problem than the other proposed models.

Finally, let us turn to the problem of describing the nucleus ${ }^{12} \mathrm{C}$ as a three-body system $\left({ }^{*}\right)$. The model was proposed by Harrington $\left(^{8}\right)$ (our model $C$ ) in Table I) and a more complete calculation, using phenomenological $\alpha-\alpha$ potentials, was made by Fulco and Wong $\left({ }^{11 a}\right)$. The papers by Leung and PARK $\left({ }^{11 b}\right)$ dealt with the same problem and Osman $\left({ }^{11 c}\right)$ explicitly calculated the Coulomb corrections to Harrington's

$\left(^{9}\right.$ D. R. Avalos, L. N. Ephee and M. A. Gregorio: Lett. Nuovo Cimento, 9, 441 (1971).

$\left({ }^{10}\right)$ H. Cohen, A. Pagnamenta and J. G. Taxlor: Nuovo Cimento, 2 A, 954 (1971).

$\left.{ }^{*}\right)$ We only refer, of course, to those calculations which use the Faddeev equations.

(11) a) J. R. Fulco and D. Y. Wong: Phys. Rev., 172, $1062(1968)$; b) C. C. H. Leung and S. C. PARK: Phys. Rev., 187, 1239 (1969); c) A. OsMan: Phys. Rev. C, 4, 302 (1971). 
model. A critical analysis of these works is not intended here; let us just comment that all these remarks, together with ours, indicate that, even if a three-body model is feasible, the actual calculation of the low-lying states of ${ }^{12} \mathrm{C}$ is a twelve-body problem rather than three-body.

One of us (MAG) is grateful to Prof. A. Salam, the International Atomic Energy Agency and UNESCO for hospitality at the International Centre for Theoretical Physics, Trieste. 